\title{
Novel Approach for Microbiome Analysis Using Bacterial Replication Rates and Causal Inference with Applications
}

\author{
Vitalii Stebliankin $^{1}$, Musfiqur Sazal $^{1}$, Camilo Valdes $^{1}$, Kalai Mathee $^{2,3}$, and Giri Narasimhan ${ }^{1,3} \bowtie$ \\ ${ }^{1}$ Bioinformatics Research Group (BioRG), Florida International University, 11200 SW 8th Street, Miami, FL 33199, USA. \\ ${ }^{2}$ Herbert Wertheim College of Medicine, Florida International University, Miami, Florida 33199, USA. \\ ${ }^{3}$ Biomolecular Sciences Institute, Florida International University, Miami, Florida 33199, USA.
}

\begin{abstract}
Motivation: Metagenomics sequencing data can be used to compute not just the relative abundance profile, but also the replication rates of every taxon in the microbiome sample. We investigate how the dynamics implied by the replication rates can be used to understand the antibiotic response in microbiomes, given the significant variation in the types of antibiotics and the types of response by different taxa. The analysis is further expanded by factoring in the resistome of the microbiomes, which can be readily profiled from the metagenomic sequence data. The fact that some antibiotics such as $\beta$-lactams target replicating cells makes it even more critical to use replication rates to analyze the antibiotic response.

Results: We introduce a novel approach for metagenomic analysis that integrates microbial community profiling, replication rate calculation, and causal structural learning to analyze the antibiotic response. First, we developed PETRI, which involves efficient cluster computation of bacterial replication rates from metagenomic sequence data. Second, we integrate the abundance profile, replication profile, resistome profile, and environmental variables to perform causality analysis. Finally, we applied the integrated analysis to the data from an infant gut microbiome study. Conclusions from our analysis are as follows: (i) Microbes tend to lower their replication rates in response to $\beta$-lactams; (ii) The presence of antibiotic resistance genes combined with the causality analysis strongly suggest that genes fos A5, $o q x A, k p n F, \operatorname{arn} A$, and $\operatorname{acr} A$ provides resistance for the taxon $K$. pneumoniae, allowing it to replicate and dominate the microbiome after the drug ticarcillinclavulanate was administered; and (iii) Human and donor milk strongly influence the resistome of the infant gut microbiome.

Availability: The pipeline for calculation of bacterial replication rates using the high performance computing framework Slurm is available from https: //biorg.cs.fiu.edu/petri/.
\end{abstract}

Bacterial Replication | Microbiome | Causality | Antibiotic resistance

Correspondence: giri@cs.fiu.edu

\section{Introduction}

Analysis of metagenomic sequencing data allows us to compute the abundance of each microbe in a microbiome $[1,2]$. Recent work has made it possible to use the same data to calculate replication rates of each microbial taxon in a sample [3-6]. Replication rates provide information on how the microbial composition may be changing, thus shedding light on microbiome dynamics. This work is motivated by a desire to understand what new insight may be deduced by this new capability. As an application, we show how replication rates can provide a nuanced understanding of the characteristics of the microbiomes and their responses to antibiotic stress.

Globally, antibiotic resistance (ABR) has emerged as one of the biggest threats to public health [7]. In the US alone, annually, over 2.8 million people get severe infections from antibiotic-resistant pathogens, resulting in about 35,000 deaths [8]. Many new antibiotics are designed to target different bacterial pathways. Yet, their efficacy wanes over time as microbes develop resistance to counter the drugs. Thus, understanding the antibiotic response of a microbiome is of utmost importance.

Antibiotics such as $\beta$-lactams target dividing cells. In some cases, bacterial taxa are known to evade the effect of $\beta$-lactam exposure by halting their division temporarily [9] and then resuming their growth at the end of the treatment [10]. Some slowly growing bacteria are known to have better survival rates under antibiotic exposure [11]. Alternatively, bacterial taxa may respond to $\beta$-lactam exposure by producing $\beta$-lactamases that hydrolyze the antibiotics providing resistance. The expression of $\beta$-lactamases is known to be proportional to bacterial growth rates [12]. Response of division rates of bacteria to antimicrobial defines its survival model [13]. Thus, the rate of replication could serve as an indicator to track ABR and to better understand bacterial defense mechanisms against antibiotics. Therefore, the growth behavior under antibiotic exposure must be investigated in the context of a microbial community, not just with bacterial isolates [11, 14].

Surprisingly, higher replication rates do not necessarily lead to a greater abundance of the bacterial taxon $[3,4,15]$. The weak correlation may be because bacte- 
rial abundance measured is a net result of replication and death. Bacterial death is not accounted for in the replication rate computation because it represents the proportion of bacteria participating in cell division $[3,16]$. By the same token, bacterial abundance, which is computed as part of standard metagenomic profiling, cannot be used as a proxy for replication rates.

Replication rates may be regulated by varying nutrient availability. In contrast, replication may be slowed down or halted in the presence of external stressors such as antibiotics. Since nutrients requirements and response to antibiotics vary with taxa, bacterial communities display a wide range of replication doubling times [17] and death rates [18] at any given time.

In this study, we include replication rate information to shed light on the dynamics within a microbiome. We use whole metagenome sequencing data to investigate the replication rates of each taxon in the microbiome to discern the impact of antibiotics and the development of resistance. We also use the theory of causal inference to study the causal relationships between the abundance and replication rates of microbial taxa, antibiotics dosages, and other clinical and environmental factors.

\section{Approach}

\subsection{Peak-To-Trough Ratio (PTR)}

Replication rates can be inferred from whole-genome DNA sequencing (mWGS) by exploiting the mechanism of bacterial cell replication [3]; circular bacterial chromosome initiate replication at the origin in both directions of the DNA strand. Thus, if bacteria are actively growing, more reads will be observed near the origin than the termination of replication. The measurement is called Peak To Trough ratio (PTR) [3] and represents the average number of replication forks per cell [16] for a given bacterial genome. Later, Brown et al. [4] implemented this method as an open-sourced software bPTR with a feature of automatic origin of replication detection.

Analysis of replication rates have been applied to several microbiome studies. For example, bacterial replication rates of some species were found to be correlated with ulcerative colitis [3], necrotizing enterocolitis (NEC) [4], and Crohn's disease [5]. Bacterial replication rates depend on the sampling site $[5,15]$ and bacterial taxonomy [4], but independent of proteome composition [19]. In addition, replication rates can provide insights into antibiotic response. Bacterial taxa can lower their replication rates in response to antibiotics $[19,20]$ and resume their growth after cessation of the exposure $[4,20,21]$. Also, average bacterial replication were shown to be correlated with quantity of ABR genes found in a sample [21].

However, the inferences drawn about microbiomes using replication rates remain fairly simplistic. One possible reason is the complexity of computing PTR. It requires mapping the sequenced reads against a ref- erence collection of genomes, which is hardly scalable for a large reference database, if the sequenced collection of reads has high sequence depth, or if we only have limited computing resources. A recent tool called FLINT demonstrates the design of scalable algorithms for metagenomic profiling by using MapReduce, streaming, and commodity resources on the cloud [22].

In this study, we developed a scalable pipeline for calculating bacterial replication rates from metagenomic samples on a cluster environment by combining bPTR tool [4] with the high-performance computing approach of FLINT [22]. Metagenome sequence data from a longitudinal study of sampled preterm infants were reanalyzed with bacterial replication rates information.

\subsection{Causal Graph}

Even though some studies were able to simultaneously measure the replication rates of several microorganisms $[6,23]$ from metagenomic samples, interactions within a microbial community were not considered in the analysis. Yet, inter-species synergies play a vital role in microbiome functioning and can create new properties not observed when studied in isolation [24]. For example, it has been shown that resident microbial communities can suppress the growth and evolution of Escherichia coli [25]. In this study, we aim to model complex causal relationships within microbial communities based on bacterial replication rates, microbial abundance, antibioticresistance genes, and miscellaneous clinical variables.

Recently, causality showed promising results to infer complex relationships (for example, colonization pattern) among different entities of a microbiome [26, 27]. Causal inference technique is useful in the classification of pathogenic and beneficial bacteria just using microbial taxa abundance information [28]. The first step of causal inference is to establish relationships among the entities, and the idea of causal structure is as follows.

Formally, we define causal structures (CS) (or causal Bayesian networks) as a class of Probabilistic Graphical Models $(P G M s)[29,30]$ where each node represents one of $n$ random variable from a set, $\mathbf{X}=\left\{X_{i}, i=1, \ldots, n\right\}$, and each edge represents a direct causal relationship. These structures are represented as a graph $G=(V, E)$, where each vertex in $V$ represents a random variable from $\mathbf{X}$, and $E$ is the set of edges. Although undirected edges are used in cases where the direction cannot be reliably determined or when both directions appear to be valid, the graph $G$ is often "manipulated" to be a Directed Acyclic Graph (DAG). Each random variable $X_{i}$ has an associated probability distribution. A directed edge in $E$ between two vertices represents direct stochastic dependencies. Therefore, if there is no edge connecting two vertices, the corresponding variables are either marginally independent or conditionally independent (conditional on the rest of the variables, or some subset thereof). The "local" probability distribution of a variable $X_{i}$ depends only on itself and its parents (i.e., the vertices with directed edges into the node $X_{i}$ ); the 
(A) PTR computational Pipeline

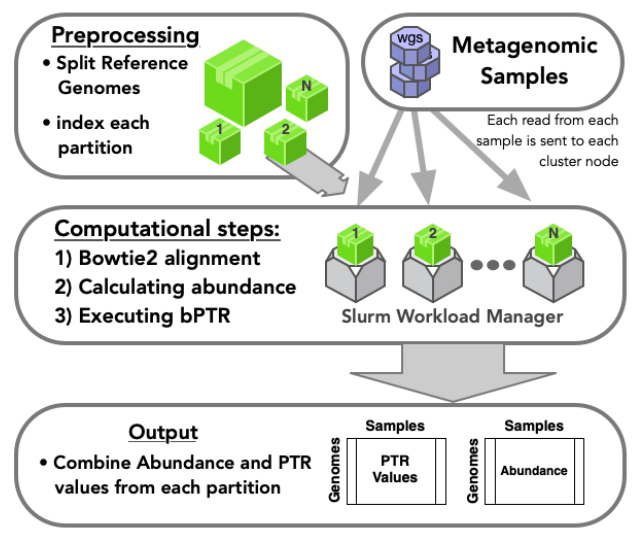

(B) ABR Gene Abundance

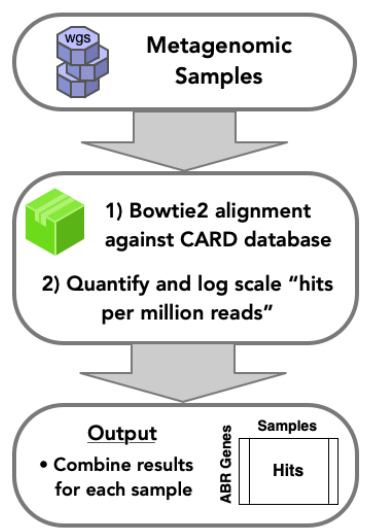

(C) Compute Causal Structural Model

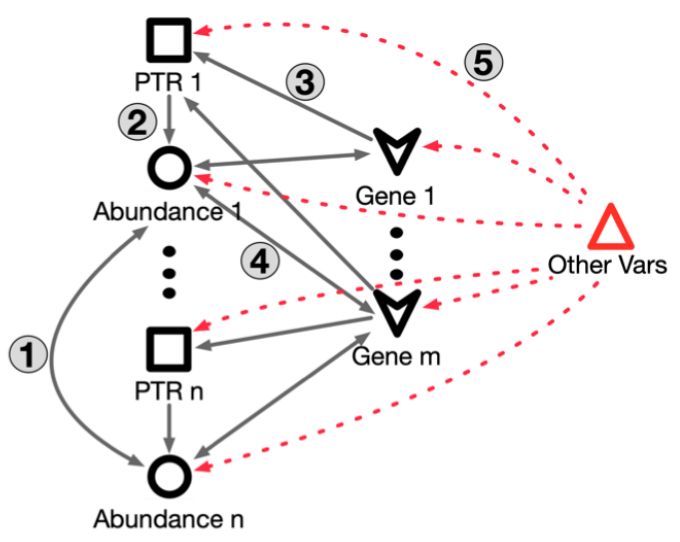

Fig. 1. Proposed approach to study relationships between replication and antimicrobial resistance A. PETRI pipeline for replication rates computation; B Pipeline for quantifying ABR genes; C. Model for causal structure where allowed edges are shown numbered 1-5. Different shapes are used for different types of nodes.

"global" probability distribution, $P(\mathbf{X})$ is the product of all local probabilities, i.e., a joint distribution [31], given by

$$
P(\mathbf{X})=\prod_{i=1}^{n} P\left(X_{i} \mid \text { Parents }\left(X_{i}\right)\right) .
$$

Note that the equation is simpler when the causal structure is sparser. Thus, an important step in our pipeline is to identify all independent pairs of random variables. More importantly, we also identify as many conditionally independent pairs as possible since these represent indirect or non-causal relationships.

All local structures in a causal structure can be classified into three sub-categories: chains, forks, and colliders. In a chain, two variables $X$ and $Y$ are conditionally independent given $Z$, if there is only one unidirectional path between $X$ and $Y$, and $Z$ is the set of variables that intercepts that path. In a fork, variable $Z$ is a "common cause" for variables $X$ and $Y$; this happens when there is no directed path between $X$ and $Y$, and they are independent conditional on $Z$. Finally, variable $Z$ is a "collider" node between $X$ and $Y$, if it is the "commoneffect". In a collider, as in the fork, there is no directed path between $X$ and $Y$. However, the difference is that $X$ and $Y$ are unconditionally independent, but become dependent when conditioned on $Z$ and any descendants of $Z$.

In general, causal models can be very complex. A pair of variables can be connected through multiple chains, forks, and colliders, making it non-trivial to determine the dependency between two arbitrary variables. Directional separation (or, just $d$-separation) is a useful concept in this context [32] because covariance terms corresponding to $d$-separated variables are equal to 0 . In a directed graph, $G$, two vertices $x$ and $y$ are $d$-connected if and only if $G$ has a collider-free path connecting $x$ and $y$. More generally, if $X, Y$ and $Z$ are disjoint sets of vertices, then $X$ and $Y$ are $d$-connected by $Z$ if and only if $G$ has a path $P$ between some vertex in $X$ and some vertex in $Y$ such that for every collider $C$ on $P$, either $C$ or a descendant $C$ is in $Z$, and no non-collider on $P$ is in $Z$. $X$ and $Y$ are $d$-separated by $Z$ in $G$ if and only if they are not $d$-connected by $Z$ in $G$. The concept of $d$-separation allows for more edges to be eliminated in a causal structure.

Meaningful causal relationships inferred from causal Bayesian networks constructed using principles of $d$ separation are more informative than association analysis. A directed edge from $X$ to $Y$ in such networks suggests that changing $X$ will imply a change in the value of $\mathrm{Y}$, if all other variables are held unchanged [33]. Therefore, with PGMs, it is possible to infer the causal impact of one variable on another, assuming that there are no hidden confounders. Even though the assumption does not hold true in reality, approximations provided by the model give valuable insights into causal relationships in a multivariate data set.

\section{Methods}

In this paper, we propose a new approach to study antibiotic resistance from microbiome sequence data. Figure 1 shows an overview of the proposed three-pronged approach. First, Figure 1 (A) presents a pipeline to efficiently calculate bacterial replication rates and relative abundance values using a high-performance computing environment. Second, (B) shows the framework to quantify ABR genes from the sequencing data. Third, we compute a causal network, where the allowed edges are as shown in the skeleton network in (C).

\subsection{PETRI: A PTR Calculation Pipeline}

To calculate bacterial replication rates for a large number of samples and reference genomes, we created a pipeline called PETRI that runs on a high-performance multiprocessor computing environment with the Slurm resource management system [34]. The pipeline consists of three steps - invoking the bowtie 2 alignment software to align the sequenced reads [35], calculating 
the abundance of each taxon in the sample using FLINT [22], and calculating growth rates of each taxon using the bPTR [4] software.

All reads were mapped by bowtie 2 to a reference genome collection containing 30,382 complete bacterial, archaeal, and viral genome sequences from the RefSeq repository [36]. Genomes were downloaded using the API offered by Kraken 2 [37].

To utilize FLINT in an optimal manner, we split the database into 64 roughly equal-sized pieces and created bowtie2 indexes as described previously [22]. As part of the preprocessing, the indexes are loaded onto the 64 different computational nodes in the cluster. Thus, each read is sent to all the 64 units, which run with their own local index of a subset of genomes from the repository.

As part of the first step in the processing pipeline, bowtie2 is used in each processor to align every read to its local index partition. Next, the alignment file is analyzed to calculate the average coverage of each genome using the following formula:

$$
C=\frac{N L}{G},
$$

where $C$ is the average coverage, $N$ is the total number of reads that align to the given genome sequence, $L$ is the average length of a metagenomic read, and $G$ is the genome sequence length. Next, we compute the relative abundance of each genomic taxon by normalizing average coverage values to add up to 1 . We filter out the list of genomes with average coverage less than 5 , which is the minimum coverage requirement for bPTR. Finally, we run the BPTR software on the set of genomes that survived the aforementioned filtering step. At the end of the processing step, for each sample, we obtain the PTR values for each genome along with the corresponding relative abundance values. The stringent filtering step is required to remove false positives from the mapping step where reads may get mapped to multiple closely related microbial strains.

Finally, we compute species-level PTR values using the following formula:

$$
P T R_{s}=\frac{\sum_{i=1}^{N} P T R_{s i} a_{s i}}{\sum_{i=1}^{N} a_{s i}},
$$

where $P T R_{s}$ is the PTR value for species $s$ with $N$ strains; $P T R_{s i}$ is the PTR value of strain $i$ of species $s$; and $a_{s i}$ is the relative abundance of strain $i$ of species $s$. Such an aggregation gives us the average PTR value for a given species $s$, and reduces the variance between values, providing more robust statistical results. Note that weighting the PTR with abundance eliminates anomalies that arise from extremely high PTR values of strains with low abundance. Additionally, it remains stable even if strains evolve over time.

The PETRI pipeline described above has many advantages over existing approaches. Our method is readily scalable. It can deal with a large number of samples, and associated sequencing reads because of the use of a cluster. In addition, our method can be scaled to deal with a huge reference genome collections. Splitting the indexes for the reference genomes database into smaller manageable pieces provides an efficient framework for the scalable computation of PTR values. The associated computation times are shorter, and the costs are meager if they are performed on a cloud service such as AWS [38]. Using more extensive genome collections allows us to measure PTR values for many more strains that may be present in the samples than currently possible with other tools, which use much smaller reference genome collections, as discussed below. Existing tools for computing PTR values such as GRiD [6] use curated reference genome collections with at most one genome per species. This is likely to limit the sensitivity of the PTR profile that is computed since strains not represented in the reference collection may have very different replication rates than the representatives. A recently developed tool called SMEG [23] deals with strain variations by finding representative reference sequence. However, we were unable to parallelize SMEG in a natural way, making it computationally slower than the tools we have used. Other tools for computing PTR values such as iRep [4] and Demic [5] use draft-quality metagenome-assembled genomes. Consequently, the computed PTR values are really coarse averages across multiple strains since reconstructed genomes often have to be dropped because of insufficient coverage or contain mosaics of multiple strains. Furthermore, metagenomic assembly and binning tasks are computationally intensive.

\subsection{Analyses of $A B R$ Genes}

DNA sequences for a list of 2624 antibiotic-resistance genes were downloaded from the Comprehensive Antibiotic Resistance Database (CARD) version 3.0.7 [39]. Metagenomic reads were then aligned against these sequences using bowtie2, allowing us to calculate "counts per million reads" for each ABR gene. Once normalized, these measures are used as a proxy for the antibiotic resistance "potential" of the metagenomic sample. Note that this potential is a substitute for the expression of the ABR genes in the sample. The CARD database also has information on the bacterial taxon from which the $\mathrm{ABR}$ gene sequence was isolated. It is important to note that it is not possible to determine (either from the metagenomic or metatranscriptomics sequence data) the specific bacterial taxon that carries the ABR gene. However, the annotation from the CARD database provides a useful prediction.

\subsection{Causal Inference}

To infer causal relationships between the variables of interest, we used the PC-stable algorithm [40] using bnlearn [41] $\mathrm{R}$ package. The multi-variate random variables used for our causal analysis include: (a) relative abundance of a taxon, (b) PTR value of a taxon, (c) dosage of maternal antibiotics administered, (d) dosage 
of infant antibiotics administered, (e) log-scaled abundance of a antibiotic resistance gene, and (f) miscellaneous environmental factors (e.g., caffeine, mother's milk, donor milk, infant birth weight, iron supplement, etc.). Note that each node of the causal network represents a multi-dimensional variable, one dimension for each sample.

To ensure biologically relevant causal structures, we restricted the set of allowed edges. Similar restrictions were introduced in Lugo-Martinez et al. [42]. As shown in Figure 1-C, the following types of edges are allowed:

Type 1: Taxon abundance $\leftrightarrow$ Taxon abundance

Type 2: PTR $\rightarrow$ abundance (of the same taxon)

Type 3: ABR gene $\rightarrow$ PTR

Type 4: Taxon abundance $\leftrightarrow$ ABR gene

Type 5: Other vars $\rightarrow$ \{Abundance, PTR, ABR gene $\}$

Edges of Type 1 between abundance values of different taxa represent microbial interactions such as cooperation or competition [43]. Edges of Type 2 suggest that a change in replication rate may change the abundance of that taxon. This paper has evidence suggesting that the presence of an ABR gene may promote the replication of the taxon that carries it (Type 3 edge). Type 4 edges suggest that higher abundance implies increased copies of the ABR gene in that sample. This may mean that increased expression may confer resistance. Finally, clinical and environmental variables are independent variables and impact all aspects of the microbiome (Type 5 edges).

All edges were filtered using a bootstrap threshold of 0.5. To keep the resulting models simple and sparse, we constructed a model using only the most abundant major bacterial species and AMR genes present in at least $5 \%$ of the samples from the study.

\subsection{Statistical Discriminant Analysis}

To determine if antibiotics significantly affected the microbial resistome, we performed Permutational Multivariate Analysis of Variance (PERMANOVA) tests [44] using the vegan $R$ package [45] using Jaccard similarity. PERMANOVA is a non-parametric test used to compare two (or more) groups of objects and test the null hypothesis that the centroids and dispersion of the groups using the distance measure are equivalent for the groups.

To visualize the differences between the two classes, we used Principal Coordinates Analysis (PCoA). PCoA is a dimensionality-reduction technique that embeds the objects in a low dimensional space so that distances are as faithfully reflected in the new space. The PCoA models were trained using ape $\mathrm{R}$ package [46].

To identify the antibiotic-resistance genes that help to discriminate between the different antibiotics, we applied the Partial least squares discriminant analysis (PLS-DA) technique for feature selection using the
MixOmics R package [47]. The PLS-DA is a supervised machine learning tool that finds the direction (i.e., principal component) that maximizes the separation among the classes. To select the set of features that contribute to the class separation the most, we used 10-fold crossvalidation and 10 repeats with tune.splsda. Statistical significance was computed using the Mann-Whitney U tests.

\subsection{Hardware}

PETRI pipeline (Section 3.1) was executed on HighPerformance Computational (HPC) resources at FIU Instructional and Research Computing Center [48]. The job submission was handled by Slurm Workload Manager version 19.05.3-2. The cluster contains 1500 Intelbased cores with High Memory Nodes ranging from 32 to 384 GB per node. All jobs were submitted to computational nodes with CentOS v.7 operating system. To be able to execute several jobs within the same computational node, we used only eight threads per one process.

Other computational steps (Sections 3.2-3.4) were performed on a server machine at FIU, castalia, with 792GB of RAM, and 48 Intel Xeon processors.

\section{Results and Discussion}

We applied our pipeline to the premature infant gut microbiome data set (BioProject ID: PRJNA301903) [49] and calculated bacterial replication rates. The data are from 401 stool samples from 84 premature infants [49]. All but two infants received antibiotic therapy within the first 24 hours. Forty-nine of the infants received additional antibiotic therapies ("Antibiotic" cohort) between 1-10 weeks of life. Remaining 35 formed the "Control" group. Each therapy consisted of one or more antibiotics (ampicillin (AMP), meropenem (MEM), ticarcillinclavulanate (TIM), gentamicin (GEN), vancomycin $(\mathrm{VAN}))$. Some treatments were occasionally mixed with Cefazolin (CFZ), Trimethoprim-Sulfamathoxazole (SXT), Clindamycin (CLI), or Cefotaxime (CTX). Stool specimens were collected at roughly 2-6 samples per infant at multiple time points between 6-156 days of life.

\subsection{Does PTR Correlate with Abundance?}

We first argue that contrary to expectations, replication rates does not positively correlate with relative abundance. In other words, increased replication does not lead to higher relative abundance values. Fig. 2-A shows the Spearman correlation between PTR and relative abundance values of the major bacterial species present in the dataset. This is also consistent with the results of Brown et al. [4]. For eight out of 25 taxa, the correlation is negative, while three species (Bifidobacterium longum, Enterococcus faecium and K. michiganensis) have a positive correlation in both premature and healthy infants.

Negative correlations between PTR and abundance values were for most part consistent in the two cohorts 
(A)

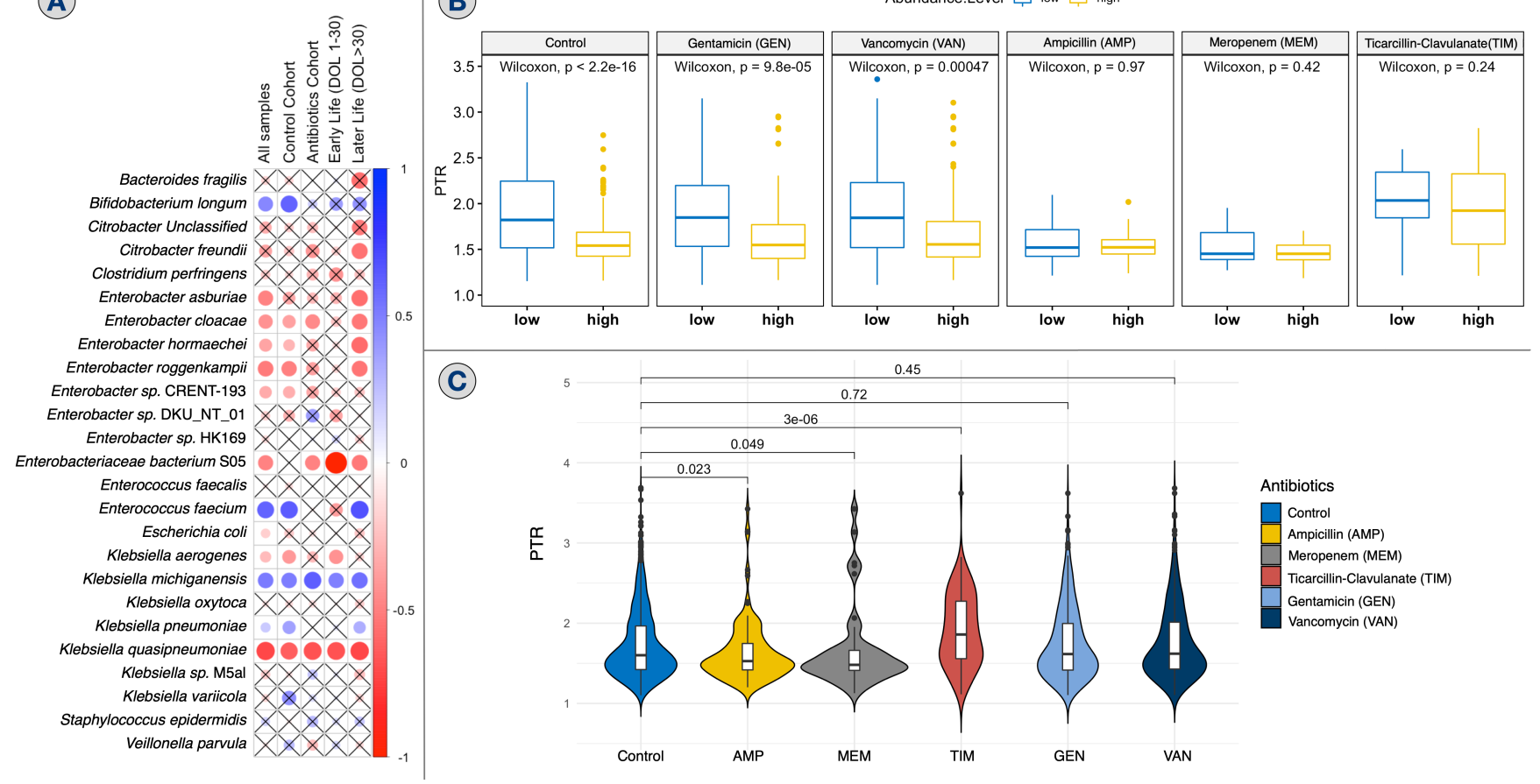

Fig. 2. A. Spearman correlation coefficients between PTR and relative abundance values of the same taxon as part of different cohorts. Species that were present in less than 20 samples were not included in this analysis. Color of the filled circles signifies the sign of the correlation (red for positive and blue for negative), while the size of the filled circles represents the absolute value of the correlation coefficient. Crossed entries represent correlations that are not significant $(p-v a l u e>0.05)$ B. Box and whisker plots of PTR values of "low" and "high" abundance bacterial taxa, broken down by the type of antibiotics administered within 48 hours prior to sampling. The "low" group includes taxa with the lowest $25 \%$ relative abundance values (values $<0.1 \%$ ), while "high" group consist of highest $25 \%$ abundant taxa (values $>4 \%$ ) C Violin plots showing distribution of PTR values in control infants and infants who recently received antibiotic treatment. Mean PTR in each treatment group was compared to control samples using the Mann-Whitney $U$ test ( $p$-values are shown in the figure).

- the "Control" cohort (infants with no additional antibiotic treatment) and the "Antibiotics" cohort (infants with additional treatment). It was also true when we sliced the data set differently, i.e., for the "Early Life" samples (first 30 days of life) as well as for "Later Life" samples (after first 30 days of life). The only inconsistencies seen were with the taxa, Enterococcus faecium spp. and Enterobacter DKU_NT_01, and with the subset of samples immediately after the administration of the $\beta$-lactam antibiotics (discussed below).

There are more cases of negative correlation (than positive) between the replication rates of taxa and their abundance. This finding implies that exhaustion of essential nutrient leads to the bacterial taxa reaching a stationary phase [50]. Figure 2-B shows PTR values of the taxa that ranked among the highest $25 \%$ (high) and lowest $25 \%$ (low) relative abundance taxa for each type of recently administered antibiotics (within 48 hours prior to sampling). Replication rates of taxa with high abundance were significantly lower than that of less abundant bacteria in the "Control" group, as well as in the cohorts that were treated with gentamicin and vancomycin.

Higher abundance did not imply lower replication when $\beta$-lactams were administered. No significant difference existed between PTR of "high" and "low" abundant species with the administration of $\beta$-lactams - MEM, AMP, and TIM. Disruption of replication is explained by the fact that $\beta$-lactams target dividing cells [51].

\subsection{How are the PTR values distributed?}

Distribution of replication rates values for each class of recently administered antibiotics is shown in Fig. 2-C. There was no significant difference between the mean PTR for the control group compared to those that were recently administered GEN or VAN. After the administration of AMP or MEM, the average PTR is lower compared to control. As mentioned earlier, this appears to be in response to the $\beta$-lactam antibiotics [10]. The lowest replication values were observed when MEM was administered, perhaps because degradation by $\beta$ lactamases appears to be least effective against this antibiotic [52]. Surprisingly, even though TIM belongs to $\beta$-lactam class, the average PTR is close to 2 after it was administered indicating that the dominant taxa were not in stationary phase after the drug exposure.

\subsection{Responses to $\beta$-lactams vis-à-vis the resistome}

If the reason for the increased replication rates of some taxa after the administration of TIM is their resistome, then it makes sense to investigate the resistome of the microbiome after the administration of TIM and the other $\beta$-lactams. PERMANOVA analysis shows that the microbial composition as well as the resistome of the infant microbiomes is significantly different for the control group and the cohorts treated with TIM and AMP/MEM (p-value <0.01; Supplementary file supplS1.xlsx - S1-A ). In Fig. 3-A the differences 

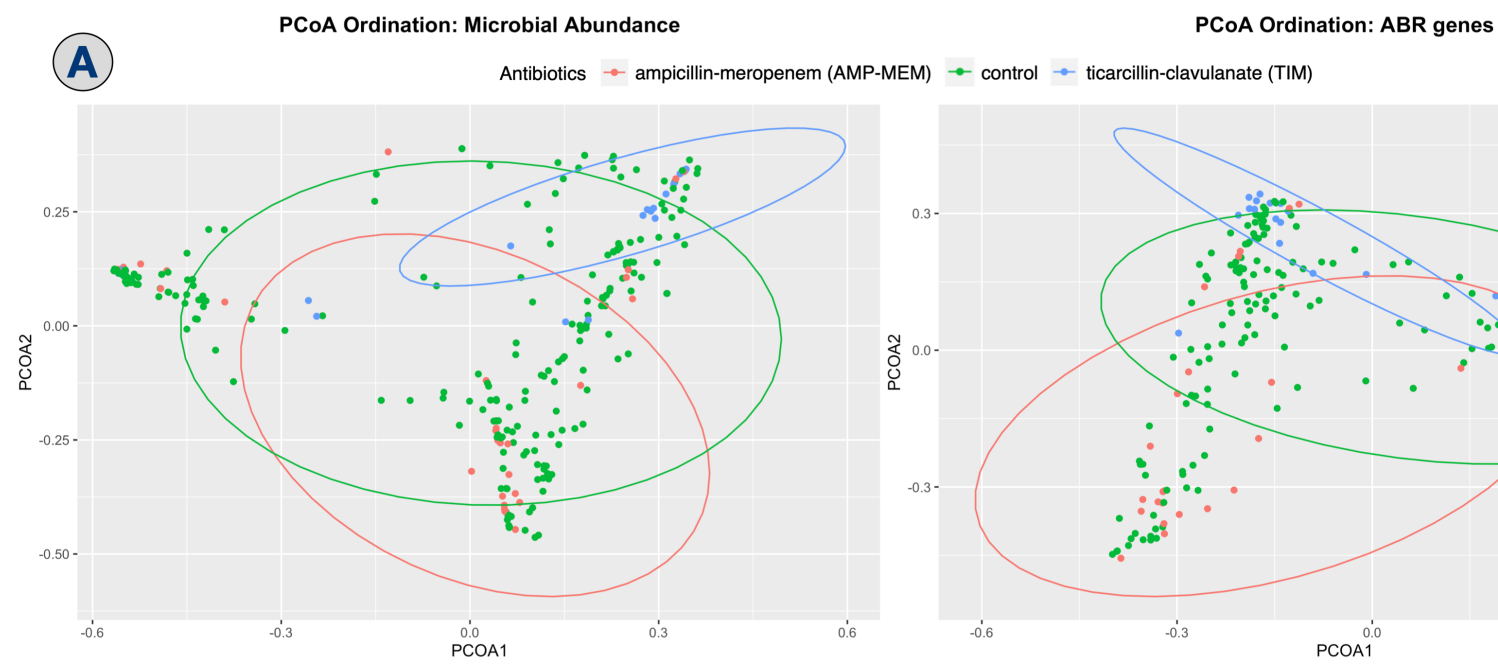

(B)

Antibiotics 追 ticarcillin-clavulanate (TIM)
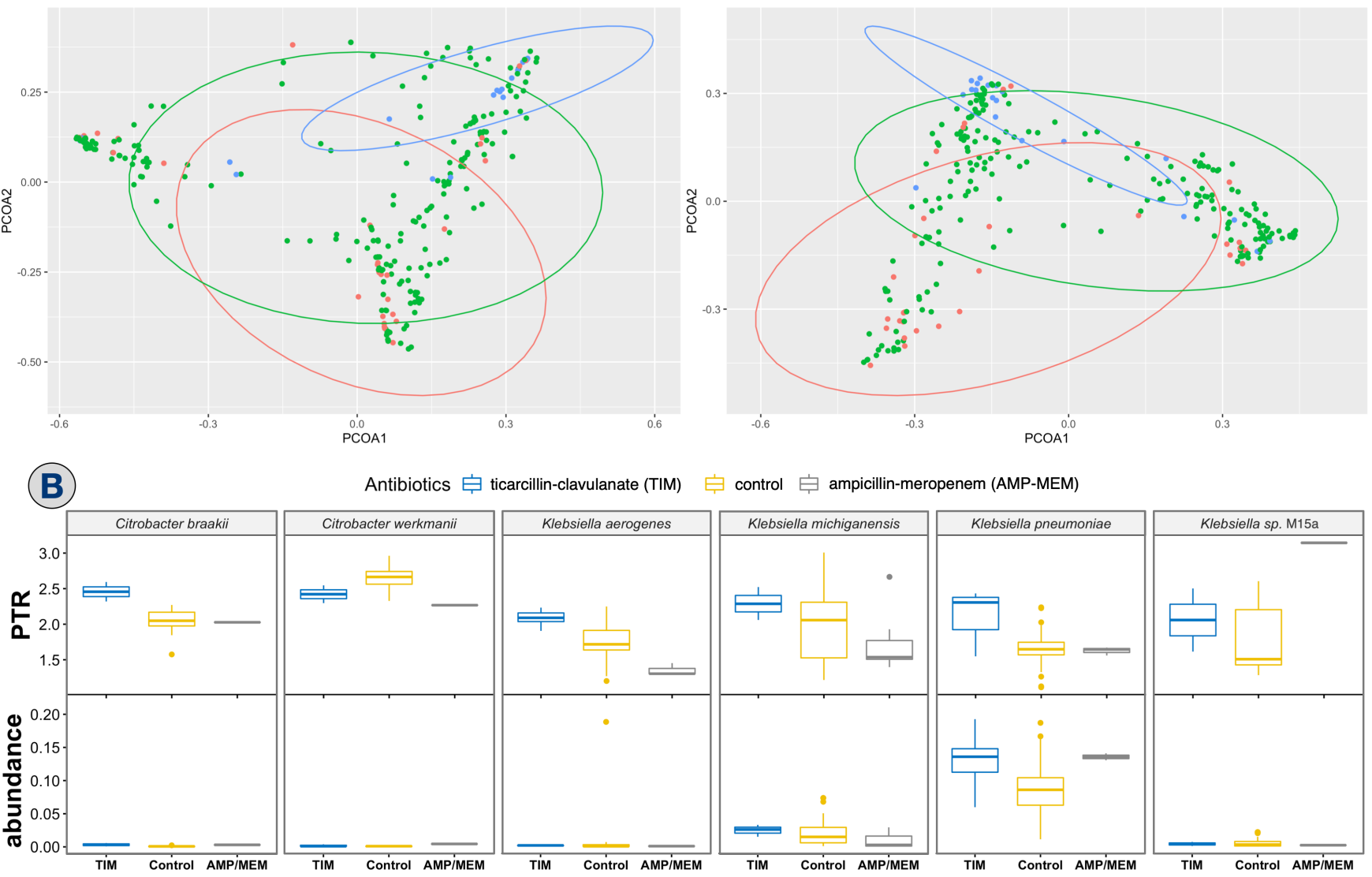

Fig. 3. A Principal coordinates analysis (PCoA) ordination based on microbial relative abundance (left) and log2-transformed "hits per million reads" of ABR genes (right). Samples represent points colored by the type of antibiotics administered within the $48 \mathrm{~h}$ prior to sampling. B Box-and-Whisker Plots of PTR (top row) and abundance (bottom row) values of bacterial taxa that were in active growth after administration of ticarcillin-clavulanate

in microbial abundance (left plot) and resistome (right plot) are shown using PCoA. Samples in the "TIM" cohort clustered more compactly suggesting a more consistent response. We note that points corresponding to the infants in the "Control" group occur closer to "TIM" cluster than the points from the "AMP/MEM" cohort. This may be because even the infants in the "Control" cohort were exposed to antibiotics during their first few days of life, possibly shaping their resistome, which gets expressed differently based on the exposure. On the other hand, the "TIM" and "AMP/MEM" cohorts favored the survival of a microbiome with different resistomes.

\subsection{Replicating taxa after antibiotics}

The taxa with the highest PTR values after administration of TIM were Citrobacter braakii, Citrobacter werkmanii, K. pneumoniae, K. aerogenes, K. michiganensis, and Klebsiella sp. M5al. The average PTR and abundance values of taxa in the active growth phase (PTR $>2$ ) after TIM administration are shown in Figure 3-B. The dominant species in the TIM cohort with a mean relative abundance of $16 \%$ was K. pneumoniae. Despite its high abundance, this species was actively growing with high PTR values, suggesting a tendency for severe dysbiosis and pathogenic dominance by this taxon. This is further evidenced by the low abundance of other potentially pathogenic species with high PTR values such as K. aerogenes and Klebsiella sp. M5al. This finding may explain why there is a high abundance of pathobionts in premature infants, an unintended consequence of antibiotic treatment $[49,53]$. Our findings suggest that the use of TIM for premature infants should be discontinued.

\subsection{Identifying $A B R$ genes of interest}

PLS-DA and Mann-Whitney U tests were used to identify specific ABR genes that contribute the most to differentiate the resistomes of the TIM from AMP/MEM cohorts. The model was trained to differentiate two cohorts: infants recently administered AMP/MEM and the TIM cohorts. To avoid overfitting the PLS-DA model [54], a 10-fold cross-validation was employed resulting in a low error rate of 0.12 . We identified $320 \mathrm{ABR}$ genes that contributed the most to differentiating the cohorts. Using the Mann-Whitney U tests and BenjaminiHochberg p-value correction, the genes of interest were reduced from 320 to 46 (see Suppl. file supplS1.xlsx S1.B) 
bioRxiv preprint doi: https://doi.org/10.1101/2020.05.21.108514; this version posted May 26, 2020. The copyright holder for this preprint (which was not certified by peer review) is the author/funder, who has granted bioRxiv a license to display the preprint in perpetuity. It is made available under aCC-BY 4.0 International license.

Table 1. ABR genes that positively correlate with replication rates and TIM treatment.

\begin{tabular}{|c|c|}
\hline ABR Gene Family & Genes correlated with average PTR* \\
\hline $\begin{array}{l}\text { K. pneumoniae } \mathrm{OKP} \\
\text { chromosomal } \beta \text {-lactamase }\end{array}$ & 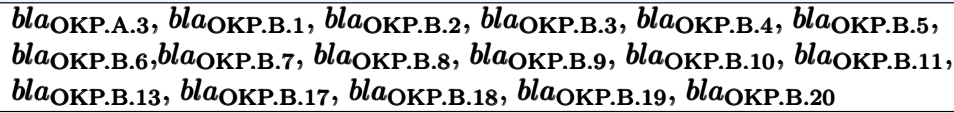 \\
\hline $\begin{array}{l}\text { Major facilitator superfamily } \\
\text { (MFS) antibiotic efflux pump }\end{array}$ & $k p n F^{*}, K$. pneumoniae $k p n G, K$. pneumoniae $k p n E$ \\
\hline $\begin{array}{l}\text { Resistance-nodulation-cell division } \\
\text { (RND) antibiotic efflux pump }\end{array}$ & $\boldsymbol{o q x} \boldsymbol{A}^{*}$, oqx $\boldsymbol{B}, \operatorname{mex} B$, smeE, $\boldsymbol{K}$. pneumoniae $\operatorname{acr} \boldsymbol{A}^{*}$-tolC \\
\hline Other & $\operatorname{arn} A^{*}$, fos $A 5^{*}$, fosA6, K. pneumoniae ompK37 \\
\hline
\end{tabular}

Of the 46 genes idenitfied in the analysis above, 30 were positively correlated with average PTR values of the TIM cohort (Table 1), of which 26 were associated with $K$. pneumoniae (shown in bold font), meaning that they were either originally isolated from it [62] or was present in at least $30 \%$ of all whole-genome shotgun assemblies available frmo NCBI for K. pneumoniae, as annotated in CARD [39].

K. pneumoniae was the most dominant species in the TIM cohort. The 26 ABR genes were associated with a resistance response to TIM, which correlates with PTR values. All the genes identified in this study as significant confer multidrug resistance to $K$. pneumoniae. The K. pneumoniae clinical isolates form three phylogenetic groups KP-I, KP-II, and KPIII $[62,63]$. K. pneumoniae are intrinsically resistant to many $\beta$-lactams due to expression of a chromosomally encoded $\beta$-lactamase constitutively conferring resistance to ampicillin, amoxicillin, carbenicillin, and ticarcillin, but not to extended-spectrum $\beta$-lactams. Three groups of bla genes also evolved parallelly bla $a_{\mathrm{SHV}}, b l a_{\mathrm{OKP}}$, and $b l a_{\mathrm{LEN}}$. The $b l a_{\mathrm{OKP}}$ family was found in group KPII [62], and bla $a_{\mathrm{OKP}-\mathrm{A}}$ and bla $a_{\mathrm{OKP}-\mathrm{B}}$ are two variants [62]. K. pneumoniae strain harboring bla $a_{\mathrm{OKP}}$ are com- mon in acute care settings and serves as a reservoir for this gene $[64,65]$. K. pneumoniae harbors many genes that confers multi-drug resistance, such as those genes encoding efflux pumps (oqxAB, kpnEF, kpnGH, acrAtolC), outermembrane porin protein (ompK37), acrAtolC (confers colistin resistance), fos $A$ (plasmid-encoded gene that confers fosfomycin resistance). The K. pneumoniae kpnEF system confers resistance to cefepime, ceftriaxone, colistin, erythromycin, rifampin, tetracycline, and streptomycin [66]. The kpnGH system confers resistance to azithromycin, ceftazidime, ciprofloxacin, ertapenem, erythromycin, gentamicin, imipenem, ticarcillin, norfloxacin, polymyxin-B, piperacillin, spectinomycin, tobramycin and streptomycin [67]. The oqxAB genes are also common in clinical isolates of Enterobacteriaceae and K. pneumoniae, conferring low to intermediated resistance to quinoxalines, quinolones tigecycline, and nitrofurantoin [68]. Loss in ompK37 in K. pneumoniae confers resistance to cefoxitin and expandedspectrum cephalosporins [69-71]. The fosA encoding fosfomycin modifying enzyme of plasmid origin are originally identified in Serratia marcesens [72, 73].

To understand which associations are causal in nature, we discuss the causality inference approach next.

Table 2. Predicted provenance of ABR genes from causal network

\begin{tabular}{|c|c|c|}
\hline Genus & Species & Predicted ABR genes * \\
\hline Bacteroides (B) & B. fragilis & $\operatorname{ermF}[55]$, серA $[56]$ \\
\hline Bifidobacterium (A) & B. longum & Bifidobacterium ileS $[57]$, mdtM, $d f r G$, Streptomyces rishiriensis par $Y$ \\
\hline Citrobacter $(\mathrm{P})$ & C. freundii & bla $_{\mathrm{CMY} .51}[58]$, bla $_{\mathrm{CMY} .66}[58]$, bla $a_{\mathrm{CMY} .67}[58]$, bla $a_{\mathrm{CMY} .59}$, isaC \\
\hline Clostridium (F) & C. perfringens & Clostridium perfringens $\boldsymbol{m p r} \boldsymbol{F}(97.09)$, ant $(6)$ Ia \\
\hline \multirow{4}{*}{ Enterobacter $(\mathrm{P})$} & E. absuriae & fosA2(87.5), bla $a_{\mathrm{MIR} .9}, b l a_{\mathrm{MIR} .10}$ \\
\hline & E. cloacae & Enterobacter cloacae acrA[59], fosA2 [60] \\
\hline & E. hormaechei & $b l a_{\mathrm{ACT} .5}(0.63)$ \\
\hline & E. roggenkampii & bla $a_{\mathrm{MIR} .10}$ \\
\hline \multirow[t]{2}{*}{ Enterococcus $(\mathrm{F})$} & E. faecalis & $\boldsymbol{d f r} \boldsymbol{E}(95.74)$, efr $\boldsymbol{B}(95.87)$ \\
\hline & E. faecium & $\boldsymbol{a a c}(\boldsymbol{6}) \boldsymbol{I i}(90.6), \boldsymbol{e f m A}(67.9), \boldsymbol{m s r C}(60.42)$ \\
\hline Escherichia $(\mathrm{P})$ & E. coli & Escherichia coli emrE (57.42), tet $B(14.53)$ \\
\hline \multirow[t]{5}{*}{ Klebsiella $(\mathrm{P})$} & K. aerogenes & $\operatorname{arn} A, \boldsymbol{F o s A 5}(86.9)$, tetB.P \\
\hline & K. oxytoca & bla $a_{\mathrm{OXY} .2 .1}[61]$, bla $\mathrm{OXY.6.3}[61], m d t K, \operatorname{ram} A, \operatorname{aph}(3) I a$ \\
\hline & K. pneumoniae & K. pneumoniae acrA $(91.56), \boldsymbol{k p n F}(99.05)$, oqxA $(92.34)$, arn $A$, fos $A 5$, bla $a_{\mathrm{SHV} .173}$, bla $a_{\mathrm{SHV} .147}$ \\
\hline & K. quasipneumoniae & bla $a_{\text {OKP.B.1 }}[62], b l a_{\mathrm{SHV} .86}$ \\
\hline & K. variicola & cdeA \\
\hline Staphylococcus $(\mathrm{F})$ & S. epidermidis & $\boldsymbol{d f r C}(98.53), \boldsymbol{m e c A}(61.76)$ \\
\hline Veillonella $(\mathrm{F})$ & V. parvula & Streptococcus agalactiae mprF \\
\hline
\end{tabular}

* In bold we show predictions that were validated by:

(\%) NCBI WGS prevalence (percentage of NCBI assemblies of predicted species to have at least one hit to a given gene);

[\#] literature claiming strong association of the given gene with predicted species;

Abbreviations: A, Actinobacteria; B, Bacteroidetes; F, Firmicutes; P, Proteobacteria. 


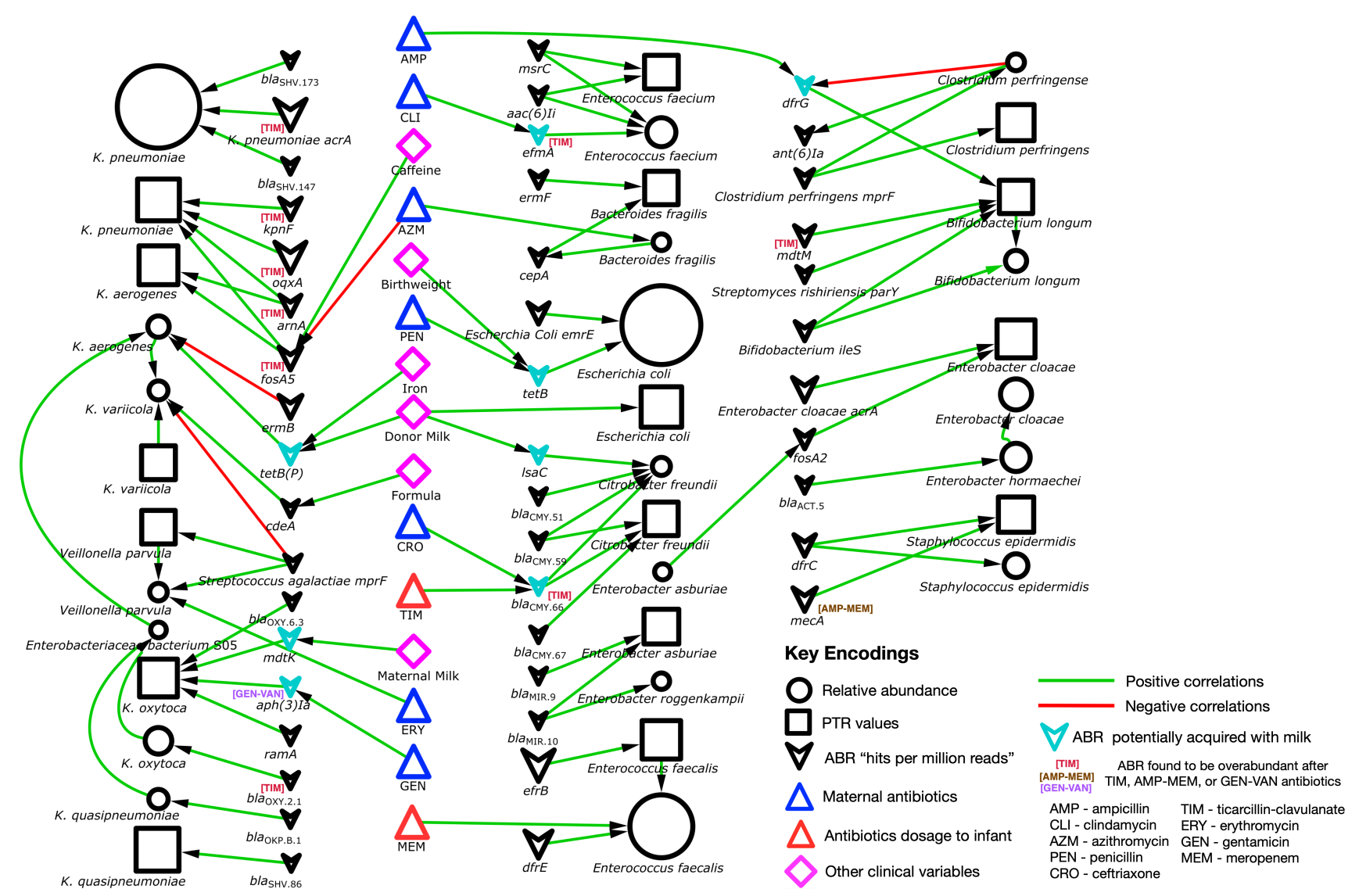

Fig. 4. Causal network for infant gut microbiome. Node sizes are proportional to the average value.

\subsection{Identifying causal relationships}

The goal of this section is to better understand antibiotic resistance response exhibited in a cohort of samples by identifying which associations or correlations are causal. The causal inferencing was performed with the same data, resulting in a graph where the nodes correspond to bacterial (relative) abundance, bacterial replication rates (PTR), abundance of ABR genes, antibiotics dosage (infant and mother), and miscellaneous environmental factors (e.g., dietary information, infant age (premenstrual and chronological), and birth-weight). Figure 4 represents the resulting causal network after removing nodes without any outgoing or incoming causal links.

\section{Predicting provenance of $A B R$ genes}

An edge directed from ABR gene to taxon abundance was surmised to mean that the taxon was the predicted source for that ABR gene. We found 48 such edges (see Table 2), of which 25 were validated as described below. In general, because of horizontal gene transfers, it is often impossible to precisely infer the an organism carrying the ABR gene. The CARD database [39] provides prevalence values giving the percentage of whole-genome shotgun assemblies of the taxon from the NCBI repository that have at least one hit to the predicted ABR gene. The database also has annotations to original studies that indicate the species from which a given gene was originally isolated. Validated genes were present in at least $50 \%$ of the assemblies or were first isolated from the predicted species.

\section{Human milk influence infant gut resistome}

Human milk (either maternal or donor) seems to have an influence on ABR genes in the infant gut microbiota. Though, we lack the mothers microbiome data, it is not far-fetched to suggest the human milk may be the source of ABR genes. The presence of ABR genes in cow milk has been demonstrated [74]. Edges out of blue triangle nodes (corresponding to antibiotics administered to the mother) suggest a clear influence on taxa abundance and $\mathrm{ABR}$ genes in the infant gut.

\section{Impact of antibiotics on bacterial taxa}

Surprisingly, antibiotic dosage shows little effect on microbial taxa. Only a few edges are coming from variables associated with antibiotic dosage administered to infants (red triangles in Fig. 4). This fact can be the implication of the model construction assumptions and restriction rules. For example, we include only major bacterial taxa that are present in at least $5 \%$ of the samples with read coverage at least $5 x$. Therefore, included species are likely to be antibiotic-resistant, which explains the lack of direct impact of antibiotics. Besides, factors such as data sparsity, high dimensionality, errors in metagenomic sequencing and read mapping, and hidden con- 
founders contribute to reduction in model sensitivity.

Nevertheless, the causal graph suggests that MEM causes an increase in abundance of Enterococcus faecalis. Enterococcus faecalis is a known early gut colonizer [75]. Enterocci are also a leading cause of hospitalacquired infections [76]. MEM is not effective against this pathogen [77]. However, antibiotic treatment can contribute to increase in the enteroccci abundance leading to reduction in the protective commensals and increase in Clostridium spp. [78].

A path from TIM $\rightarrow$ bla $a_{\mathrm{CMY} .66} \rightarrow$ Citrobacter (both PTR and abundance), suggests that Citrobacter freundii express $b l a_{\mathrm{CMY}} .66$ encoding $\beta$-lactamases that inactivate TIM. The presence of this gene in Citrobacter freundii has been previously shownn [79]. This finidng argues that without knowing apriori the source of a gene, an educational guess could be made through our proposed novel approach. The presence of ABR strain of Citrobacter freundii is of major concern among pediatric population as it has been known to caused serious neonatal infections [80].

\section{Impact of ABR genes on replication and abundance}

The causal analysis sheds light on which ABR genes played the most critical role in the survival of some pathogens against antibiotic stress. Even though, according to the causal network, antibiotics dosage directly affects only a few ABR genes, we still can identify those that were most active against a particular drug. Using a Mann-Whitney U test, we identified genes that were significantly abundant after specific treatment. Such genes are indicated in colored font within square brackets in Fig. 4.

For example, bla OXY.2.1 is annotated with [TIM] meaning that number of hits to the sequence were significantly higher in infants with TIM administered within 48 hours prior to sampling. Edge leading from the gene to abundance of $K$. oxytoca suggest its role in allowing this pathogen to thrive.

Most importantly, our analysis suggests how $K$. pneumonia becomes dominant and actively replicating after the administration of TIM antibiotics. Fig. 4 indicates that five of ABR genes overabundant in TIM group have direct edges to PTR or abundance of this species. In particular, we hypothesize that fosA5, oqxA, kpnF, $\operatorname{arn} A$, and $\operatorname{acr} A$ are possibly contributing to an increase in growth of $K$. pneumonia.

ABR genes are particularly influential when edges to both the abundance and PTR nodes of bacterial taxa. For example, difC helps Staphylococcus epidermidis to grow in presence of AMP and MEM.

A previous study [21] found that MFS genes are associated with increased replication for any bacterial taxa. In our study, we show that the influence of ABR genes on bacterial growth is not bound to specific gene families, but on the species that carries it and the type of antibiotics. Causal links between an ABR gene and a PTR node does not necessarily imply direct involvement in increasing regulation, which may be the result of nutrients availability or other indirect causes.

\section{Conclusion}

In this work, we propose a new computational pipeline called PETRI, the first software package available for distributed computation of bacterial replication rates (PTR), which uses a reference-based approach with all available complete microbial genomes from the RefSeq database. Our approach shows how the replication rates computed by PETRI can be used in the construction of a model for causal structural learning to infer potential cause effect relationships between bacterial replication, bacterial abundance, and environmental factors.

When integrated into any whole metagenome sequence analysis, we obtain relative abundance values along with replication rates of the bacteria in the microbiome. Even though replication rate data is readily available, it is not usually computed. Here we apply this enhanced analysis pipeline on a publicly available infant gut microbiome dataset, and show that it can shed light on microbiome dynamics and provide new insights on antibiotic resistance.

Our results confirm that in general, higher replication does not imply growth in abundance. In fact, taxa with high abundance on average have low replication rates (i.e., in stationary phase).

Replication rates are found to be lower after the administration of $\beta$-lactams like ampicillin and meropenem, perhaps because they target dividing cells. However, some pathogens were actively replicating in the presence of $\beta$-lactam compound, TIM. In fact, pathogen $K$. pneumoniae was the most abundant and actively growing after the administration of this antibiotic. K. pneumoniae has been shown to be pathogenic and able to increase the mortality rate in preterm infants by 30 percent [81]. The causal network suggests that genes fosA5, oqxA, kpnF, acrA, and $\operatorname{arn} A$ had a causal effect on the abundance and/or replication rate. Therefore, K. pneumoniae has the necessary resistome to thrive after TIM treatment, and this drug should be avoided for premature infants.

Other interesting conclusions from the causal analysis include the influence of mother or donor milk on the resistome present in the infant's gut. Thus, human milk should be tested for the presence of ABR genes [82].

Our methods show that even if an antibiotic resistance gene is novel, or in the absence of the CARD database, our method is still likely to identify its role in the microbiome.

The results of causal analysis need to be tested in the laboratory. Errors in inference may be caused by small sample size, errors in sequencing, errors in mapping reads to the correct genomes, errors in abundance matrix, presence of undocumented or new bacterial taxa, inability to measure known or hidden confounders related to the diet and environment of the mother and 
bioRxiv preprint doi: https://doi.org/10.1101/2020.05.21.108514; this version posted May 26, 2020. The copyright holder for this preprint (which was not certified by peer review) is the author/funder, who has granted bioRxiv a license to display the preprint in perpetuity. It is made REFERENCES available under aCC-BY 4.0 International license.

infant, and much more. Nevertheless, we set the stage for a novel approach to study microbiome dynamics from studies with limited time points.

\section{Acknowledgements}

The authors thank the members of the Bioinformatics Research Group (BioRG) at FIU for valuable feedback and comments.

\section{Funding}

This work was partly supported by the National Institute of Health (1R15AI128714-01).

\section{References}

1. Nicola Segata, Levi Waldron, Annalisa Ballarini, Vagheesh Narasimhan, Olivier Jousson, and Curtis Huttenhower. Metagenomic microbial community profiling using unique cladespecific marker genes. Nature methods, 9(8):811, 2012

2. Derrick $E$ Wood and Steven $L$ Salzberg. Kraken: ultrafast metagenomic sequence classification using exact alignments. Genome biology, 15(3):R46, March 2014

3. T. Korem, D. Zeevi, J. Suez, A. Weinberger, T. Avnit-Sagi, M. Pompan-Lotan, E. Matot, G. Jona, A. Harmelin, N. Cohen, A. Sirota-Madi, C. A. Thaiss, M. Pevsner-Fischer, R. Sorek, R. J. Xavier, E. Elinav, and E. Segal. Growth dynamics of gut microbiota in health and disease inferred from single metagenomic samples. Science, 349(6252):1101-1106, 2015. ISSN 0036-8075, 1095-9203.

4. Christopher T Brown, Matthew R OIm, Brian C Thomas, and Jillian F Banfield. Measurement of bacterial replication rates in microbial communities. Nature Biotechnology, 34(12):12561263, December 2016. ISSN 1087-0156, 1546-1696.

5. Yuan Gao and Hongzhe Li. Quantifying and comparing bacterial growth dynamics in multiple metagenomic samples. Nature Methods, 15(12):1041-1044, December 2018. ISSN 15487091, 1548-7105.

6. Akintunde Emiola and Julia Oh. High throughput in situ metagenomic measurement of bacterial replication at ultra-low sequencing coverage. Nature Communications, 9(1), December 2018. ISSN 2041-1723.

7. Mark Woolhouse, Catriona Waugh, Meghan Rose Perry, and Harish Nair. Global disease burden due to antibiotic resistance-state of the evidence. Journal of Global Health, 6, 06 2016.

8. CDC. Antibiotic Resistance Threats in the United States. Atlanta, GA: U.S. Dept. of Health and Human Services;. 2019.

9. A Tomasz. The mechanism of the irreversible antimicrobial effects of penicillins: How the $\beta$-lactam antibiotics kill and lyse bacteria. Annual Review of Microbiology, 33(1):113-137, 1979 .

10. Gerald R Donowitz and Gerald L Mandell. Beta-lactam antibiotics. New England Journal of Medicine, 318(7):419-426, 1988.

11. RM Cozens, E Tuomanen, W Tosch, O Zak, J Suter, and A Tomasz. Evaluation of the bactericidal activity of beta-lactam antibiotics on slowly growing bacteria cultured in the chemostat. Antimicrobial Agents and Chemotherapy, 29(5):797-802, 1986.

12. BIRGITTA Engberg and $\mathrm{K}$ Nordström. Replication of $r$-factor $r 1$ in scherichia coli $k-12$ at different growth rates. Journal of bacteriology, 123(1):179-186, 1975.

13. Loïc Marrec and Anne-Florence Bitbol. Resist or perish: fate of a microbial population subjected to a periodic presence of antimicrobial. PLOS Computational Biology, 16(4) e1007798, 2020.

14. J. N. Anderl, J. Zahller, F. Roe, and P. S. Stewart. Role of Nutrient Limitation and Stationary-Phase Existence in Klebsiella pneumoniae Biofilm Resistance to Ampicillin and Ciprofloxacin. Antimicrobial Agents and Chemotherapy, 47(4):1251-1256, April 2003. ISSN 0066-4804, 1098-6596.

15. Matthew R. Olm, Christopher T. Brown, Brandon Brooks, Brian Firek, Robyn Baker, David Burstein, Karina Soenjoyo, Brian C. Thomas, Michael Morowitz, and Jillian F. Banfield. Identical bacterial populations colonize premature infant gut, skin, and oral microbiomes and exhibit different in situ growth rates. Genome Research, 27(4):601-612, April 2017. ISSN 1088-9051, 1549-5469.

16. H. Bremer and G. Churchward. An examination of the Cooper-Helmstetter theory of DNA replication in bacteria and its underlying assumptions. Journal of Theoretical Biology, 69 (4):645-654, December 1977. ISSN 00225193.

17. Beth Gibson, Daniel J Wilson, Edward Feil, and Adam Eyre-Walker. The distribution of bacterial doubling times in the wild. Proceedings of the Royal Society B: Biological Sciences, 285(1880):20180789, 2018.

18. Michael L Pace. Bacterial mortality and the fate of bacterial production. Hydrobiologia, 159 (1):41-49, 1988.

19. Christopher T Brown, Weili Xiong, Matthew R Olm, Brian C Thomas, Robyn Baker, Brian Firek, Michael J Morowitz, Robert L Hettich, and Jillian F Banfield. Hospitalized premature infants are colonized by related bacterial strains with distinct proteomic profiles. Mbio, 9(2): e00441-18, 2018.

20. Hyunmin Koo, Joseph A Hakim, David K Crossman, Ranjit Kumar, Elliot J Lefkowitz, and Casey D Morrow. Individualized recovery of gut microbial strains post antibiotics. NPJ biofilms and microbiomes, 5(1):1-6, 2019.
21. Sumayah F Rahman, Matthew R Olm, Michael J Morowitz, and Jillian F Banfield. Machine learning leveraging genomes from metagenomes identifies influential antibiotic resistance genes in the infant gut microbiome. MSystems, 3(1):e00123-17, 2018.

22. Camilo Valdes, Vitalii Stebliankin, and Giri Narasimhan. Large scale microbiome profiling in the cloud. Bioinformatics, 35(14):i13-i22, 07 2019. ISSN 1367-4803.

23. Akintunde Emiola, Wei Zhou, and Julia Oh. Metagenomic growth rate inferences of strains in situ. Science Advances, 6(17):eaaz2299, 2020.

24. Karoline Faust, J Fah Sathirapongsasuti, Jacques Izard, Nicola Segata, Dirk Gevers, Jeroen Raes, and Curtis Huttenhower. Microbial co-occurrence relationships in the human microbiome. PLoS Comp Bio, 8(7):e1002606, 2012.

25. Michael Baumgartner, Florian Bayer, Katia R Pfrunder-Cardozo, Angus Buckling, and Alex $\mathrm{R}$ Hall. Resident microbial communities inhibit growth and antibiotic-resistance evolution of escherichia coli in human gut microbiome samples. PLoS Biology, 18(4):e3000465, 2020.

26. Musfiqur Rahman Sazal, Kalai Mathee, Daniel Ruiz-Perez, Trevor Cickovski, and Giri Narasimhan. Inferring directional relationships in microbial communities using signed Bayesian networks. BMC Bioinformatics, In Press, 2020.

27. Musfiqur Rahman Sazal, Daniel Ruiz-Perez, Trevor Cickovski, and Giri Narasimhan. Inferring relationships in microbiomes from signed Bayesian networks. In 8th Intl. Conf. on Computational Advances in Bio and Medical Sciences (ICCABS), page 1. IEEE, 2018.

28. Musfiqur Rahman Sazal, Vitalii Stebliankin, Kalai Mathee, and Giri Narasimhan. Causal inference in microbiomes using intervention calculus. bioRxiv, 2020.

29. Judea Pearl. Probabilistic reasoning in intelligent systems: networks of plausible inference. Elsevier, 2014.

30. Daphne Koller and Nir Friedman. Probabilistic graphical models: principles and techniques. MIT press, 2009.

31. Marco Scutari. Bayesian network constraint-based structure learning algorithms: Paralle and optimised implementations in the bnlearn r package. arXiv preprint arXiv:1406.7648, 2014.

32. Dan Geiger, Thomas Verma, and Judea Pearl. d-separation: From theorems to algorithms In Machine Intelligence and Pattern Recognition, volume 10, pages 139-148. Elsevier, 1990.

33. Clark Glymour, Kun Zhang, and Peter Spirtes. Review of causal discovery methods based on graphical models. Frontiers in Genetics, 10, 2019.

34. Andy B Yoo, Morris A Jette, and Mark Grondona. Slurm: Simple linux utility for resource management. In Workshop on Job Scheduling Strategies for Parallel Processing, pages 44-60. Springer, 2003.

35. Ben Langmead and Steven Salzberg. Fast gapped-read alignment with Bowtie 2. Nature methods, 9:357-9, 032012

36. Nuala A O'Leary, Mathew W Wright, J Rodney Brister, Stacy Ciufo, Diana Haddad, Rich McVeigh, Bhanu Rajput, Barbara Robbertse, Brian Smith-White, Danso Ako-Adjei, et al. RefSeq database at NCBI: current status, taxonomic expansion, and functional annotation. Nucleic acids research, 44(D1):D733-D745, 2016.

37. Derrick E Wood, Jennifer Lu, and Ben Langmead. Improved metagenomic analysis with kraken 2. Genome biology, 20(1):257, 2019.

38. Amazon.com Inc,. Amazon Web Services. https://aws.amazon.com/. Accessed: 2020-04 13.

39. Brian P Alcock, Amogelang R Raphenya, Tammy TY Lau, Kara K Tsang, Mégane Bouchard, Arman Edalatmand, William Huynh, Anna-Lisa V Nguyen, Annie A Cheng, Sihan Liu, et al. Card 2020: antibiotic resistome surveillance with the comprehensive antibiotic resistance database. Nucleic acids research, 48(D1):D517-D525, 2020.

40. Diego Colombo and Marloes H Maathuis. Order-independent constraint-based causal structure learning. The Journal of Machine Learning Research, 15(1):3741-3782, 2014.

41. Marco Scutari and Jean-Baptiste Denis. Bayesian Networks with Examples in R. Chapman and Hall, Boca Raton, 2014. ISBN 978-1-4822-2558-7, 978-1-4822-2560-0.

42. Jose Lugo-Martinez, Daniel Ruiz-Perez, Giri Narasimhan, and Ziv Bar-Joseph. Dynamic interaction network inference from longitudinal microbiome data. Microbiome, 7(1):54, 2019.

43. Mitch Fernandez, Juan D Riveros, Michael Campos, Kalai Mathee, and Giri Narasimhan. Microbial" social networks". BMC genomics, 16(11):S6, 2015.

44. Marti J Anderson. A new method for non-parametric multivariate analysis of variance. Austral ecology, 26(1):32-46, 2001.

45. Jari Oksanen, Roeland Kindt, Pierre Legendre, Bob O'Hara, M Henry H Stevens, Maintainer Jari Oksanen, and MASS Suggests. The vegan package. Community ecology package, 10:631-637, 2007

46. Emmanuel Paradis and Klaus Schliep. ape 5.0: an environment for modern phylogenetics and evolutionary analyses in R. Bioinformatics, 35(3):526-528, 2019

47. Florian Rohart, Benoit Gautier, Amrit Singh, and Kim-Anh Lê Cao. mixOmics: An R package for 'omics feature selection and multiple data integration. PLoS comput biol, 13(11): e1005752, 2017.

48. Instructional and Research Computing Center (IRCC). http://ircc.fiu.edu. Accessed: 202004-17.

49. Molly K. Gibson, Bin Wang, Sara Ahmadi, Carey-Ann D. Burnham, Phillip I. Tarr, Barbara B. Warner, and Gautam Dantas. Developmental dynamics of the preterm infant gut microbiota and antibiotic resistome. Nature Microbiology, 1(4):16024, March 2016. ISSN 2058-5276.

50. Roberto Kolter, Deborah A Siegele, and Antonio Tormo. The stationary phase of the bacterial life cycle. Annual review of microbiology, 47(1):855-874, 1993.

51. KOK-FAI KONG, Lisa Schneper, and Kalai Mathee. Beta-lactam antibiotics: from antibiosis to resistance and bacteriology. Apmis, 118(1):1-36, 2010.

52. Claudine M Baldwin, Katherine A Lyseng-Williamson, and Susan J Keam. Meropenem. Drugs, 68(6):803-838, 2008.

53. Sourabh Dutta, Meenakshi Ganesh, Pallab Ray, and Anil Narang. Intestinal colonization among very low birth weight infants in first week of life. Indian pediatrics, 51(10):807-809, 2014.

54. Daniel Ruiz Perez and Giri Narasimhan. So you think you can PLS-DA? BMC Bioinformatics, In Press, 2020

55. Achille Aime Kangaba, Filiz Yarimcam Saglam, Hrisi Bahar Tokman, Mert Torun, and 
bioRxiv preprint doi: https://doi.org/10.1101/2020.05.21.108514; this version posted May 26, 2020. The copyright holder for this preprint (which was not certified by peer review) is the author/funder, who has granted bioRxiv a license to display the preprint in perpetuity. It is made available under aCC-BY 4.0 International license.

Muzeyyen Mamal Torun. The prevalence of enterotoxin and antibiotic resistance genes in clinical and intestinal bacteroides fragilis group isolates in turkey. Anaerobe, 35:72-76, 2015.

56. Marc B Rogers, Tamara K Bennett, Catherine M Payne, and C Jeffrey Smith. Insertional activation of cepa leads to high-level beta-lactamase expression in bacteroides fragilis clinical isolates. Journal of bacteriology, 176(14):4376-4384, 1994.

57. Fausta Serafini, Francesca Bottacini, Alice Viappiani, Enrico Baruffini, Francesca Turroni, Elena Foroni, Tiziana Lodi, Douwe van Sinderen, and Marco Ventura. Insights into physiological and genetic mupirocin susceptibility in bifidobacteria. Appl. Environ. Microbiol., 77 (9):3141-3146, 2011

58. UniProt Consortium. Uniprot: a worldwide hub of protein knowledge. Nucleic acids research, 47(D1):D506-D515, 2019.

59. David Keeney, Alexey Ruzin, and Patricia A Bradford. Rama, a transcriptional regulator, and acrab, an rnd-type efflux pump, are associated with decreased susceptibility to tigecycline in enterobacter cloacae. Microbial drug resistance, 13(1):1-6, 2007.

60. H Xu, V Miao, W Kwong, R Xia, and J Davies. Identification of a novel fosfomycin resistance gene (fosa2) in enterobacter cloacae from the salmon river, canada. Letters in applied microbiology, 52(4):427-429, 2011.

61. Cindy Fevre, Mehdi Jbel, Virginie Passet, François-Xavier Weill, Patrick AD Grimont, and Sylvain Brisse. Six groups of the oxy $\beta$-lactamase evolved over millions of years in klebsiella oxytoca. Antimicrobial agents and chemotherapy, 49(8):3453-3462, 2005.

62. Cindy Fevre, Virginie Passet, François-Xavier Weill, Patrick AD Grimont, and Sylvain Brisse. Variants of the klebsiella pneumoniae okp chromosomal beta-lactamase are divided into two main groups, okp-a and okp-b. Antimicrobial agents and chemotherapy, 49(12):5149-5152, 2005.

63. Sylvain Brisse and Jan Verhoef. Phylogenetic diversity of klebsiella pneumoniae and klebsiella oxytoca clinical isolates revealed by randomly amplified polymorphic dna, gyra and parc genes sequencing and automated ribotyping. International journal of systematic and evolutionary microbiology, 51(3):915-924, 2001.

64. Andrea Endimiani, John M DePasquale, Sandra Forero, Federico Perez, Andrea M Hujer, Daneshia Roberts-Pollack, Paul D Fiorella, Nancy Pickens, Brandon Kitchel, Aida E Casiano-Colon, et al. Emergence of bla kpc-containing klebsiella pneumoniae in a longterm acute care hospital: a new challenge to our healthcare system. Journal of antimicrobial chemotherapy, 64(5):1102-1110, 2009.

65. Hesna Yigit, Anne Marie Queenan, Gregory J Anderson, Antonio Domenech-Sanchez, James W Biddle, Christine D Steward, Sebastian Alberti, Karen Bush, and Fred C Tenover. Novel carbapenem-hydrolyzing $\beta$-lactamase, $\mathrm{kpc}-1$, from a carbapenem-resistant strain of klebsiella pneumoniae. Antimicrobial agents and chemotherapy, 45(4):1151-1161, 2001.

66. Vijaya Bharathi Srinivasan and Govindan Rajamohan. Kpnef, a new member of the klebsiella pneumoniae cell envelope stress response regulon, is an smr-type efflux pump involved in broad-spectrum antimicrobial resistance. Antimicrobial agents and chemotherapy, 57(9):4449-4462, 2013

67. Vijaya Bharathi Srinivasan, Bharat Bhushan Singh, Nitesh Priyadarshi, Neeraj Kumar Chauhan, and Govindan Rajamohan. Role of novel multidrug efflux pump involved in drug resistance in klebsiella pneumoniae. PLoS One, 9(5), 2014.

68. Federico Perez, Susan D Rudin, Steven H Marshall, Peter Coakley, Liang Chen, Barry N Kreiswirth, Philip N Rather, Andrea M Hujer, Philip Toltzis, David Van Duin, et al. Oqxab, a quinolone and olaquindox efflux pump, is widely distributed among multidrug-resistan klebsiella pneumoniae isolates of human origin. Antimicrobial agents and chemotherapy, 57(9):4602-4603, 2013

69. Santiago Hernández-Allés, Vicente J Benedí, Luis Martínez-Martínez, Álvaro Pascual, Alicia Aguilar, Juan M Tomás, and Sebastián Albertí. Development of resistance during antimicrobial therapy caused by insertion sequence interruption of porin genes. Antimicrobial agents and chemotherapy, 43(4):937-939, 1999

70. Antonio Doménech-Sánchez, Santiago Hernández-Allés, Luis Martínez-Martínez, Vicente J Benedí, and Sebastián Albertí. Identification and characterization of a new porin gene of klebsiella pneumoniae: its role in $\beta$-lactam antibiotic resistance. Journal of bacteriology, 181(9):2726-2732, 1999

71. Luis Martinez-Martinez, Santiago Hernández-Allés, Sebastian Albertí, Juan M Tomás, Vicente $\mathrm{J}$ Benedi, and George A Jacoby. In vivo selection of porin-deficient mutants of klebsiella pneumoniae with increased resistance to cefoxitin and expanded-spectrumcephalosporins. Antimicrobial Agents and Chemotherapy, 40(2):342-348, 1996.

72. Lynn L Silver. Fosfomycin: mechanism and resistance. Cold Spring Harbor perspectives in medicine, 7(2):a025262, 2017.

73. JUAN EVARISTO Suárez and MARIA CARMEN Mendoza. Plasmid-encoded fosfomycin resistance. Antimicrobial agents and chemotherapy, 35(5):791, 1991

74. Adrienn Gréta Tóth, István Csabai, Eszter Krikó, Dora Tozser, Gergely Maróti, Árpád V Patai, László Makrai, Géza Szita, and Norbert Solymosi. Raw milk for human consumption may carry antimicrobial resistance genes. bioRxiv, page 853333, 2019.

75. Michael S Gilmore, Francois Lebreton, and Willem van Schaik. Genomic transition of enterococci from gut commensals to leading causes of multidrug-resistant hospital infection in the antibiotic era. Current opinion in microbiology, 16(1):10-16, 2013

76. Bradley D Jett, Mark M Huycke, and Michael S Gilmore. Virulence of enterococci. Clinica microbiology reviews, 7(4):462-478, 1994

77. GL Drusano, H Lode, and JR Edwards. Meropenem: clinical response in relation to in vitro susceptibility. Clinical microbiology and infection, 6(4):185-194, 2000.

78. Krista Dubin and Eric G Pamer. Enterococci and their interactions with the intestinal microbiome. Bugs as Drugs: Therapeutic Microbes for the Prevention and Treatment of Disease, pages $309-330,2018$

79. Dimitria A Mathys, Dixie F Mollenkopf, Sydnee M Feicht, Rachael J Adams, Amy L Albers, David M Stuever, Susan V Grooters, Gregory A Ballash, Joshua B Daniels, and Thomas E Wittum. Carbapenemase-producing enterobacteriaceae and aeromonas spp. present in wastewater treatment plant effluent and nearby surface waters in the us. PloS one, 14(6), 2019.

80. FC Lin, WILLIAM F Devoe, CHARLOTTE Morrison, JOSEPH Libonati, PATRICIA Powers, RJ Gross, B Rowe, EBENEZER Israel, and Jr JG Morris. Outbreak of neonatal citrobacter diversus meningitis in a suburban hospital. The Pediatric infectious disease journal, 6(1): 50-55, 1987.

81. P Dorota, A Chmielarczyk, L Katarzyna, M Piotr, L Jan, R Renata, and W Jadwiga. Klebsiella pneumoniae in breast milk-a cause of sepsis in neonate. Arch Med, 9(1), 2017.

82. Nancy E Wight. Donor human milk for preterm infants. Journal of Perinatology, 21(4): 249-254, 2001. 